\title{
Progress in the Treatment of Edema After Surgery for Mixed Hemorrhoids
}

\author{
Xiaolan Hua', Yu Zhao', Huairun Zuo', Jinghua Liang2* \\ 1Shaanxi University of Traditional Chinese Medicine, Xianyang 712000, China \\ ${ }^{2}$ Anorectal Department, Shenzhen Hospital of Traditional Chinese Medicine, Shenzhen 518000, China \\ *Corresponding author: Jinghua Liang, liangjinghua0918@126.com
}

\begin{abstract}
Edema is a common postoperative complication in mixed hemorrhoids. With the development of medical treatment, surgical improvement has greatly reduced surgical trauma to the body, but the issue of postoperative edema still exists. In order to explore the treatment progress of edema after surgery for mixed hemorrhoids, literatures on integrated traditional Chinese and western medicine for postoperative edema in hemorrhoids have been reviewed in hope to better understand the complication and provide reference for relevant clinical studies in the future.
\end{abstract}

Keywords: Mixed hemorrhoids; Postoperative edema; Treatment methods; Traditional Chinese and western medicine

Publication date: November 2021; Online publication: November 30, 2021

\section{Introduction}

Mixed hemorrhoids is a common anorectal disease. The common manifestations of the disease are bleeding, pain, prolapsed hemorrhoids, anal itching, and other clinical symptoms. Many patients opt for surgical treatment after clinical consultation. However, being an open and contaminated wound postoperatively, the occurrence of edema at the site of the wound is common in the healing process. This has always been a problem for many anorectal surgeons. In mixed hemorrhoids, postoperative edema would not only cause anal swelling and pain, but also lead to slow surgical wound healing ${ }^{[1]}$. The treatment progress of integrated traditional Chinese and western medicine for postoperative edema in hemorrhoidal disease is discussed in this paper.

2. Traditional Chinese and western medicine in the treatment of postoperative edema in mixed hemorrhoids

\subsection{Treatment with western medicine}

Oral western medicine such as diosmin and Mai Ling tablets usually has anti-inflammatory properties, improves perianal circulation, and promotes lymphatic reflux. In a study ${ }^{[2]}$, Mayinglong ointment was applied for the patients in the control group, and the patients in the experimental group were treated with diosmin in addition to the former. It was found that the total efficiencies were $78 \%$ and $94 \%$ for the control group and experimental group, respectively. The effect was significantly better for the patients who received combination therapy compared to those in the control group. In another study ${ }^{[3]}$, comparing the control group (oral hemorrhoid Ning tablets) with the experimental group (oral Mai Ling tablets), postoperative edema resolved significantly faster in the experimental group compared to its counterpart.

Mixed hemorrhoids at the anal ring result in a large damage area, and the skin elasticity around the anus deteriorates. After surgery, anal stenosis caused by scar contracture along with blocked local lymphatic 
drainage and poor blood circulation result in edema. Zhang Qiang, He Chunmei, and Lu Jingen conducted a research, where anal expansion of 2 min was performed at about $3.3 \mathrm{~cm}$ after anesthesia in a group of patients with mixed hemorrhoids ${ }^{[4]}$, while in another group, the patients were routinely treated. The edema score of the wound margin was observed in the two groups and compared, in which the group that underwent anal enlargement had a significantly lower score than that of the control group. In another study, Du Guangxin also studied on anal expansion for postoperative edema ${ }^{[5]}$. In the study, on the third day after surgery, the patients were advised to avoid exerting pressure, and using the forefinger and middle finger to expand the anus, on the seventh day, the patients were observed for any signs of postoperative edema. It was noted that the patients in the experimental group had less postoperative edema compared to the patients in the conventional treatment group. The intervention significantly improved the patients' postoperative comfort, shortened the recovery cycle, and reduced the medical costs.

Physical therapy for postoperative edema uses microwave, red light, photon therapy instrument, and other instruments through direct irradiation of the human body. They directly act on blood vessels and subcutaneous tissues, accelerating the removal of inflammatory factors and improving the circulation of blood as well as local lymphatic drainage. In a research conducted by Zhong Chaoxiang ${ }^{[6]}$, photon therapy instrument and Jinma lotion were used to observe the degree of wound edema, and it was found that the efficiency of the combination was as high as 97.5\%. In another study ${ }^{[7]}$, Wang Min and Zhong Zhengming divided 106 patients into 2 groups: an observation group (diosmin and mayinglong hemorrhoid cream combined with photon therapy) and a control group (oral diosmin and mayinglong hemorrhoid cream). The patients in both the groups were observed, and it was noted that the edema on day- 4 and day- 6 of the observation group was significantly lower than that of the control group.

\subsection{Treatment with TCM}

Traditional Chinese medicine treatment requires reasoning through TCM syndrome differentiation and treatment. It should be guided by the "four diagnoses of traditional Chinese medicine." In a research conducted by Gao Linghui ${ }^{[8]}$, by observing the clinical effect of an agent (astragalus, crown ginseng, Dangshen, angelica, yam, white, white peony, hemp, firewood, tangerine, licorice, etc.) on various complications in the treatment of mixed hemorrhoids, the results showed that the agent helped in resolving the pain and edema in the treatment of mixed hemorrhoids along with promoting wound healing. There were no obvious adverse reactions during the trial, indicating that the agent is a safe and effective drug. In another study ${ }^{[9]}$, Zhitong Rushen decoction was used in patients with edema after hemorrhoidectomy. It was noted that the regression time of edema was shorter in this group of patients compared to those in the potassium permanganate group. In addition, the edema score was lower and the pain experienced as well as total efficiency were significantly better compared to the potassium permanganate group. This shows that shen soup is conducive to the postoperative recovery of patients and helps to the relieve discomfort symptoms.

Traditional Chinese medicine fumigation sitz bath involves the use of a corresponding dosage of Chinese herbal medicine. After hydration, the skin of the affected part is fumigated, so as to achieve the purpose of the drug. In a study, a homemade Chinese medicine sitz bath (25 grams of yellow bai, 20 grams of dandelion, 20 grams of bitter ginseng, 15 grams of frankincense, 15 grams of 15 myrrh, 15 grams of belvedere fruit, 15 grams of "snake bed," and 15 grams of ground elm) was used for patients with postoperative edema ${ }^{[10]}$. In contrast to the potassium permanganate sitz bath, the homemade sitz bath was significantly better in resolving the edema. In another study, Hu Qi compared the effect of using Jinxuan hemorrhoid fumigation bath (purslane, xuan powder, alum, honeysuckle, and mustard) with potassium permanganate sitz bath. After five days, no edema was observed among the patients in the study group ${ }^{[11]}$. 
This shows that Jinxuan hemorrhoid fumigation bath can significantly improve postoperative anal edema, quality of life, and patients' satisfaction.

Other than that, Chinese herbal medicine can also be made into powder, diluted with water in proportion, and then applied to the affected area. As the drug is directly applied on the wound, it accelerates absorption. In Zhang Haoliang's dissertation ${ }^{[12]}$, by comparing the use of rhubarb and manro powder with $50 \%$ magnesium sulfate, it was found that the efficacy of the former is better than the latter. In another study, Shuangbaisan (big yellow, side cypress leaves, yellow cypress, Zeeland, mint in 2:2:1:1:1 ratio) was applied to the affected edematous site after treatment ${ }^{[13]}$. Compared to $50 \%$ magnesium sulfate wet compress, the effect of the former was better.

In terms of acupuncture therapy, Wang Wei treated several patients (control group) with thunder-fire moxibustion for 20 to 30 minutes on selected strong points, while the treatment group was treated on this basis in addition to placing the tip of the needle into the skin on both forearms. The patients were guided to press on the needle 3 4 times a day for 1 2 minutes in the ward for 5 days. The needles were replaced every 48 hours. From day-4 onwards, the edema of the patients in the treatment group significantly improved compared to the control group ${ }^{[14]}$.

In terms of traditional Chinese medicine ointment, a study was carried out to determine the effect of fifty swelling paste (25 grams of angelica daidica, 30 grams of pentaploid, 10 grams of saponin, 20 grams of rhubarb, and vaseline) on postoperative edema in hemorrhoidal disease. The paste was applied to the edema site once a day. Compared to the application of magnesium sulfate, the treatment rate was $93.3 \%$, $56.7 \%$, and $23.3 \%$, respectively, indicating that the fifty swelling paste helps to resolve anal edema after hemorrhoidectomy ${ }^{[15]}$.

In terms of suppositories, a study was done to observe the improvement of anal edema by using indomethacin notoginseng borneol suppository. There was no significant change on day 3; however, there was a significant improvement on days 7,11 , and $16(P<0.01)^{[16]}$. This shows that the suppository drug plays a significant role in alleviating postoperative edema. By comparing a homemade suppository with an anal suppository, Fan Yazhao found that the pain, bleeding, and edema experienced by the patients treated with the homemade suppository were significantly lower than that of the anal suppository group ${ }^{[17]}$.

\section{Conclusion}

There are various treatment methods which have good curative effect for postoperative edema, but each treatment method has its own advantages and disadvantages. Certain measures can be taken before the surgery to prevent local edema from occurring. This includes the prevention of postoperative diarrhea, constipation, and other problems, which can aggravate local edema. In addition, a timely treatment for edema should also be taken into consideration. With the development of traditional Chinese medicine in China and the gradual standardization of drugs and surgical techniques, the use of traditional Chinese medicine for treatment has good prospects. The use of integrated traditional Chinese and western medicine in the treatment of edema after hemorrhoid surgery is worth deeper investigation.

\section{Disclosure statement}

The authors declare that there is no conflict of interest.

\section{References}

[1] Hu J, Feng Q, 2017, Data Mining Traditional Chinese Medicine Fumigation Method for Edema After Mixed Hemorrhoids. Shaanxi TCM, 38(7): 967-968. 
[2] Zhou C, 2016, Effect of Site and Ma Yinglong Hemorrhoid Ointment on Patients with Mixed Hemorrhoids with Edema. Contemporary Medicine Theory Cluster, 14(8): 179-180.

[3] Lei J, 2020, Clinical Efficacy of Applying Meiling Tablets After Mixed Hemorrhoidectomy. Drug Evaluation, 17(21): 60-62.

[4] Zhang Q, He C, Lu J, 2017, Efficacy Evaluation of mixed Hemorrhoids Based on "Disease" Anal Expansion Method Combined with Chinese Medicine Fumigation to Prevent Complications After Ring Mixed Hemorrhoids. Chinese General Practice, 20(S2): 332-334.

[5] Du G, 2016, Effect of Anal Expansion on Preventing Edema After Mixed Hemorrhoid Surgery. Journal of Practical Gynecological Endocrinology (Electronic Edition), 3(16): 109.

[6] Zhong C, Yao Q, Zhang Q, et al., 2016, Photon Therapy Instrument Combined with Jinma Lotion for Mixed Hemorrhoids After Edema. Chinese Journal of Surgery of Integrated Traditional Chinese and Western Medicine, 22(4): 399-401.

[7] Wang M, Zhong Z, 2015, Effect of Wound Edema and Pain After Diosamine and Mixed Hemorrhoids. Laser Magazine, 36(10): 162-164.

[8] Gao L, Wang Y, 2017, Clinical Study of Complications After Mixed Hemorrhoids. Liaoning Journal of Traditional Chinese Medicine, 44(5): 955-958.

[9] Zhang Z, Zou Y, Wang Y, et al., 2018, Clinical Observation of TCM Smoked Sitting Bath for Complications After Mixed Hemorrhoids. Chinese TCM Emergency, 27(2): 315-317.

[10] Hu Q, 3026, Clinical Effect of Jinxuan Hemorrhoid Department on Treating Edema and Promoting Wound Healing. The World Journal of Integrated Traditional Chinese and Western Medicine, 11(12): 1724-1727.

[11] Zhang H, 2016, Clinical Study of External Application to Edema After Hemorrhoid Surgery. Xinjiang Medical University.

[12] Xiao J, Li Z, Wang R, et al., 2018, Clinical Observation of Edema for Mixed Hemorrhoids. Chinese Folk Therapy, 26(12): 19-21.

[13] Wang W, 2018, Effect of Press Needle and Thunder Fire Moxibustion on Postoperative Wound Pain and Edema in Patients with Mixed Hemorrhoids. Inner Mongolia Traditional Chinese Medicine, 37(12): 69-70.

[14] An X, 2016, Clinical Effect of Edema After Mixed Hemorrhoids. Heilongjiang University of Traditional Chinese Medicine.

[15] Bai J, Lu B, Zheng K, et al., 2018, Diosiine Combined with Indopexin Tripatch Was Treated for Anal Edema After Mixed Hemorrhoid. Journal of Jilin Medical College, 39(1): 12-14.

[16] Fan Y, Ruan C, Jiang H, et al., 2019, Clinical Study of Haemorrhoid Complications. Chinese Basic Medicine, 26(2): 220-223.

[17] Luo Y, Zhang X, Zhu L, 2016, Smoking Therapy Promotes Healing After Infectious Wound. Jilin Traditional Chinese Medicine, 36(4): 372-375. 